\title{
The role of lineage specifiers in pancreatic ductal adenocarcinoma
}

\author{
Soledad A. Camolotto ${ }^{1}$, Veronika K. Belova ${ }^{1}$, Eric L. Snyder ${ }^{1,2}$ \\ ${ }^{1}$ Huntsman Cancer Institute, University of Utah, Salt Lake City, UT, USA; ${ }^{2}$ Department of Pathology, University of Utah, Salt Lake City, UT, USA \\ Contributions: (I) Conception and design: All authors; (II) Administrative support: None; (III) Provision of study materials or patients: None; (IV) \\ Collection and assembly of data: All authors; (V) Data analysis and interpretation: All authors; (VI) Manuscript writing: All authors; (VII) Final \\ approval of manuscript: All authors. \\ Correspondence to: Eric L. Snyder. Department of Pathology, University of Utah, Salt Lake City, UT 84112, USA. Email: eric.snyder@hci.utah.edu.
}

\begin{abstract}
Over the last decade, multiple genomics studies have led to the identification of discrete molecular subtypes of pancreatic ductal adenocarcinoma. A general theme has emerged that most pancreatic ductal adenocarcinoma (PDAC) can be grouped into two major subtypes based on cancer cell autonomous properties: classical/pancreatic progenitor and basal-like/squamous. The classical/progenitor subtype expresses higher levels of lineage specifiers that regulate endodermal differentiation than the basal-like/ squamous subtype. The basal-like/squamous subtype confers a worse prognosis, raising the possibility that loss of these lineage specifiers might enhance the malignant potential of PDAC. Here, we discuss several of these differentially expressed lineage specifiers and examine the evidence that they might play a functional role in PDAC biology.
\end{abstract}

Keywords: Lineage specifier; molecular subtype; transcription factor

Submitted Mar 13, 2018. Accepted for publication Apr 20, 2018.

doi: 10.21037/jgo.2018.05.04

View this article at: http://dx.doi.org/10.21037/jgo.2018.05.04

\section{Introduction}

Pancreatic ductal adenocarcinoma (PDAC) is the most common cancer arising from the pancreas. PDAC is believed to develop from morphologically distinct noninvasive precursor lesions; namely, pancreatic intraepithelial neoplasia (PanIN), intraductal papillary mucinous neoplasm (IPMN) and mucinous cystic neoplasm (MCN), in order of decreasing frequency (1). Despite its low incidence, PDAC is currently the fourth leading cause of cancer mortality in the United States and represents a major health burden. The low survival rate and poor prognosis are largely due to its aggressive and metastatic nature, disappointing response to therapy, and late diagnosis as a consequence of lack of effective screening strategies and absence of specific symptoms at early stages $(2,3)$.

Thus, there is an urgent need to understand PDAC at the molecular and genomic level in order to improve overall survival. Although KRAS mutations are almost universal in PDAC, this is an otherwise genetically heterogeneous disease that displays a complex mutational landscape. TP53, $C D K N 2 A$ and SMAD4 are the most commonly mutated oncogenic drivers after $K R A S$, but a growing number of other low-prevalence mutated genes have been identified in various genomic studies (4).

Gene expression profiling has been instrumental for subtype classification in cancer. Variance in gene expression patterns among cancer subtypes reflects fundamental differences in tumors at the molecular level. Identification of tumor subtypes by transcriptomic analysis has biological, prognostic and predictive value, can enable patient stratification and reveal potential therapeutic vulnerabilities (1). Several groups have used transcriptomic analysis, alone or in combination with other modalities, to identify biologically and clinically distinct subsets of PDAC. For example, Moffitt et al. identified two major subtypes (classical and basal-like) (5), Collisson et al. identified three (quasimesenchymal, classical, and exocrine like) (6), and Bailey et al. identified four [squamous, pancreatic progenitor, aberrantly differentiated endocrine exocrine 
Table 1 Lineage specifier expression in PDAC subtypes, effect in pancreatic cancer progression and prognostic value

\begin{tabular}{lccll}
\hline Transcription factor & Classical/progenitor subtype & Basal-like/squamous subtype & Effect & Prognostic value \\
\hline FoxA1/FoxA2 & + & - & Dichotomous & Undetermined \\
HNF4 $\alpha$ & + & - & Tumor suppressor & Yes \\
HNF1 $\alpha$ & + & - & Dichotomous & Yes \\
PDX1 & + & - & Dichotomous & Yes \\
\hline
\end{tabular}

PDAC, pancreatic ductal adenocarcinoma.

(ADEX), and immunogenic] (7). Most recently, the Cancer Genome Atlas Research Network reported an integrated genomic, transcriptomic and proteomic analysis of an additional 150 PDAC samples (8). The authors also revisited the three prior genomic analyses and found significant overlap between the squamous (7) and basal-like (5) subtypes and also between the pancreatic progenitor (7) and the classical subtypes described in Collisson et al. (6) and Moffitt et al. (8). The authors argue that PDAC can fundamentally be classified into two major subtypes based on cancer cell autonomous properties: basal-like/squamous and classical/pancreatic progenitor. They further suggest that additional subtypes identified to date (exocrine-like, ADEX and immunogenic) reflect either contributions from non-neoplastic tissue or less common subtypes not included in their cohort.

One major difference between these two major subtypes is the relative levels of several lineage specifiers, i.e., transcription factors that regulate endodermal development and differentiation. Classical/progenitor subtypes express high levels of a transcriptional network pivotal for pancreatic endodermal determination, including genes such as FOXA2, FOXA3, HNF4A, HNF4G, HNF1A, HNF1B and $P D X 1$ (7). In contrast, the basal-like/squamous subtype exhibits loss of endodermal identity by hypermethylation and downregulation of these lineage specifiers and concomitant upregulation of pathways driven by MYC, $\Delta$ Np63 and TGF- $\beta(7)$.

The basal-like/squamous subtype confers poor prognosis, with a median survival significantly shorter than the classical/progenitor subtype $(5,7)$. This raises the possibility that these lineage specifiers are not merely markers of PDAC subtype, but might regulate the malignant potential of this disease. Here we review the literature on the role of relevant lineage specifiers in normal pancreas and PDAC pathogenesis, with a specific focus on those that are highly expressed in the classical/progenitor subtype relative to the basal/squamous subtype (Table 1).

\section{FoxA1 and FoxA2}

The winged helix transcription factors FoxA1 and FoxA2 together control the development and morphogenesis of both the exocrine and endocrine pancreas. FoxA1 and FoxA2 are expressed in the foregut endoderm prior to pancreatic development and persist in all islet and acinar cells into adulthood (9). Pancreata of Foxa1 and Foxa2-null mice showed diminished PDX1 expression, intense pancreatic hypoplasia, hyperglycemia, impaired acinar and islet cell content, and subsequent death. Endoderm-specific genetic deletion of Foxa2 in mice provoked severe hypoglycemia, impaired $\alpha$-cell differentiation and early death (9). Interestingly, FoxA1 and FoxA2 are expressed in the foregut endoderm prior to multiple relevant lineage specifiers, such as PDX1, HNF1 $\beta$, PTF $1 \alpha, \mathrm{HNF} 4 \alpha$, etc. (10). In fact, it has been shown that FoxA1 and FoxA2 participate in the transcriptional activation of $P d x 1$ (11), as well as $H N F 4 A, H N F 1 A, H N F 1 B$ and $H N F 4 G$ in the pancreas and other endodermal organs (12-14), suggesting a role as upstream transactivators in the transcriptional hierarchy.

Although FoxA1 and FoxA2 proteins are pivotal regulators of normal development of different endoderm-derived organs such as pancreas, liver, lungs and prostate (15), their involvement in cancer remains largely unexplored. Nevertheless, some progress has been made elucidating the role of these important pioneer factors in pancreatic carcinogenesis. For example, Mishra and Guda found that FOXA1 (as well as HNF4A and HNF1A) are hypermethylated in primary PDAC tumors versus normal samples in their analysis of previously published TCGA studies (16). An additional report identified FoxA1 as one of the transcription factors that characterize the classical PDAC gene signature described by Collisson et al. (6). The authors showed that FoxA1 levels and enhancerassociated binding is dramatically downregulated in highgrade compared to low-grade human PDAC cell lines (17). Moreover, an immunohistochemical analysis of 25 
human PDAC specimens showed that FoxA1 and FoxA2 were expressed in normal pancreatic epithelium, acinar to ductal metaplasia, PanINs and well differentiated invasive PDACs. In contrast, FoxA1 and FoxA2 were downregulated in poor to undifferentiated PDACs (18). In human PDAC cell lines, FoxA1 and FoxA2 impose a transcriptional program that sustains an epithelial phenotype while antagonizing the epithelial to mesenchymal transition (EMT), in part by positively regulating E-cadherin expression (18). However, this study did not directly address the impact of altered FoxA1/2 expression on the overall tumorigenicity of these cell lines.

With regard to mechanisms by which FoxA1 and FoxA2 levels might be regulated in PDAC, two studies have shown that FoxA1 and/or FoxA2 are downregulated during TGF $\beta$-induced EMT in PDAC cell lines $(18,19)$. In the course of elucidating the role of GATA6 in pancreatic oncogenesis, FOXA2 was shown to be a GATA6 target gene and to positively correlate with GATA6 levels in PDAC samples (20). ChIP-Seq data in this study also suggest that GATA6 and FoxA2 may cooperatively regulate a proepithelial/anti-mesenchymal gene expression program in PDAC (20). Finally, miR-199a can inhibit FOXA2 expression in human PDAC cells by directly binding its $3^{\prime}$ UTR (21). FoxA2 suppression in a panel of human PDAC cell lines (using siRNA, CRISPR, and miR199a expression) increased cellular growth, invasion, migration and colony formation, as well as tumor growth in xenografts (21).

Although more work needs to be done, the above studies are generally consistent with a model in which loss of FoxA1/2 activity might impair an endodermal differentiation program and enhance tumorigenesis. However, a recent report shows that the situation is likely to be more complex than this simple model would suggest. Specifically, Roe et al. used an innovative organoid culture system to identify genes implicated in metastasis during PDAC progression (22). Using ChIP-Seq for histone $\mathrm{H} 3$ lysine 27 acetylation (H3K27ac, a marker of active enhancers and promoters) on a panel of organoids derived from primary and metastatic tumors, they found that the metastatic process is promoted by a dramatic reprogramming of enhancer activity (22). FoxA1, in cooperation with GATA5, was identified as a central driver of enhancer reprogramming, activating a foregut differentiation program and promoting a more invasive and anchorage-independent behavior in vitro as well as an increased metastatic potential in vivo without inducing EMT (22). Thus, it appears that FoxA activity can either promote or restrain tumorigenesis, depending on the specific context. Additional studies are clearly needed to obtain a better understanding of this dichotomy.

\section{HNF40}

The nuclear receptor superfamily member HNF4 $\alpha$ is a master regulator of epithelial differentiation in multiple tissues, including the gastrointestinal tract and liver (23-26). In the pancreas, HNF4 $\alpha$ is vital for $\beta$ cell function through the regulation of several genes involved in metabolismsecretion coupling (27) and glucose stimulated insulin secretion (28). Mutations in this gene have been associated with Mature-Onset Diabetes of the Young 1 (MODYI), a monogenic autosomal dominant non-insulin-dependent diabetes mellitus type I (29). More recently, it was suggested that loss of HNF4 $\alpha$ function alters endoplasmic reticulum $\mathrm{Ca}^{2+}$ homeostasis and insulin release in $\beta$-cells of patients with MODYI (30).

Although HNF4 $\alpha$ has been implicated in the pathogenesis of different malignancies (31) and the regulation of multiple biological processes that influence tumor progression, such as proliferation (32-34), apoptosis (35), metabolism $(36,37)$ and inflammation $(38,39)$, little is known about its role in PDAC. Kim et al. successfully developed a model to study early pancreatic cancer progression by generating induced pluripotent stem cells (iPSC) from primary human PDAC (40). This PDAC-iPSC line generates endodermal ductal structures reminiscent of PanIN and further progresses to invasive pancreatic cancer in vivo when implanted in mice (40). Protein profiling from PanIN organoid cultures revealed a network centered in HNF4 $\alpha$. Using IHC, the authors showed that HNF4 $\alpha$ expression is absent or barely detectable in normal human or mouse pancreatic ducts, weak in PanIN1, moderate in PanIN2, and stronger and uniform in PanIN3 and well differentiated PDAC (40). Consistent with published genomics studies, HNF $4 \alpha$ is essentially undetectable in undifferentiated or poorly differentiated PDAC (40). This study suggested that the dynamic expression of $\mathrm{HNF} 4 \alpha$ and its target genes at different stages of PDAC progression might become a useful diagnostic and prognostic biomarker of pancreatic malignancy (40).

Additional studies have shown that HNF4 $\alpha$ levels may have an impact on the malignant potential and therapeutic response of PDAC. EMT in pancreatic cancer has been linked to a greater proclivity for migration and invasion, gain of stemness and increased anticancer treatment resistance (41). Viotti et al. carried out an siRNA screen to identify epigenetic factors responsible for EMT 
in pancreatic cancer, demonstrating that the histone methyltransferase SUV420H2 acts as an orchestrator of epithelial/mesenchymal states (42). SUV420H2 induces silencing of key drivers of epithelial cell identity, such as HNF $4 \alpha$ and FoxA1, and its attenuation diminishes invasion, migration, stem cell characteristics, and chemoresistance in human pancreatic cancer cells (42). Additionally, the authors showed correlation between EMT and increased SUV420H2 expression during the pathological progression of PDAC (42).

Based on the Collisson et al. PDAC subclassification and the suggested differential chemotherapy response between the classical and quasimesenchymal subtypes (6), Noll et al. interrogated the contribution of different cytochrome P450 (43) enzymes, which play a major role in xenobiotic metabolism, in drug resistance in PDAC (44). In this study, HNF4 $\alpha$ and NR1I2 were responsible for mediating basal and drug-induced expression of CYP3A5, respectively, and the conferred drug resistance (44). Thus, interfering with the regulation of CYP3A5 mechanism by these transcription factors offers a potential mechanism to overcome basal and acquired drug resistance in PDAC. Interestingly, HNF4 $\alpha$ can be pharmacologically targeted by small molecule antagonists recently described in the preclinical setting $(45,46)$, offering the possibility of suppressing HNF $4 \alpha$ transcriptional activity.

Finally, HNF $4 \alpha$ levels can be modulated by specific drugs, which may enable therapeutic strategies that alter the differentiation state of PDAC cells. In a mouse model of IPMN/PDAC driven by oncogenic KRAS ${ }^{\text {G12D }}$ and deletion of $\mathrm{Brg} 1$ (47), the bromodomain inhibitor JQ1 concomitantly reduces tumor burden and attenuates expression of HNF4 $\alpha$ and PDX1 while increasing the expression of mature murine duct markers, possibly through reactivation of a cellular differentiation program (47). Treatment of the human PDAC cell lines PANC-1 and CFPAC- 1 with cantharidin, a vesicant produced by beetles, as well as its derivative norcantharidin, impaired proliferation and downregulated putative stem cell markers (including $\mathrm{HNF} 4 \alpha, \mathrm{MYC}$, CD44, SOX9, HMGA2, EPCAM, EPCAM and SOX2) while strengthening the cytotoxicity of gemcitabine and erlotinib in co-treatment experiments (48). These results show potential therapeutic promise for the eradication of pancreatic CSC, which are a small subset of malignant cells capable of self-renewal and are thought to play a critical role in regulating pancreatic cancer progression, metastasis, and drug resistance (48). Additionally, a recent study identified a PDAC side population in 52 human PDAC resected tumors (49). Gene expression analysis by microarray revealed not only an enrichment in pancreatic CSC markers and a chemoresistance-associated phenotype but also upregulation of molecular networks associated with cellular growth and proliferation, cell-cell signaling and amino acid metabolism, which includes $\mathrm{HNF} 4 \alpha$ (49).

\section{HNF1a}

$\mathrm{HNF} 1 \alpha$ is a homeodomain transcription factor specifically involved in later islet cell formation and function. $H N F 1 \alpha$ mutation is one of the most prevalent causes of transcription factor autosomal dominant diabetes. It regulates the expression of genes involved in glucose, amino acid, and tricarboxylic acid metabolism and is necessary for mitochondrial function in pancreatic islet cells $(43,50,51)$.

Given the important role of HNF $1 \alpha$ in pancreas development, several reports have explored its function in the pathogenesis of pancreatic malignancy. Evaluation of a large cohort of pancreatic cancer patients identified HNF $1 \alpha / \beta$ and their predicted targets to be globally downregulated in PDAC samples compared to normal pancreas tissues (52). Using transcriptome analysis on a series of human cell lines, PDAC and normal pancreas samples, Hoskins et al. found gene expression changes associated with upregulation of genes involved in EMT as well as repression of transcription factors that participate in pancreatic development and homeostasis, where HNF1 $\alpha$ and HNF $1 \alpha$-regulated networks were the most significantly affected (53). Furthermore, HNF1 $\alpha$ expression is lost in a subset of human PDAC samples and tissue microarrays as detected by immunohistochemistry (53).

HNF $1 \alpha$ may serve as a prognostic biomarker and predictor of therapy response in PDAC (54). Biopsies of two independent cohorts of resected patients were stratified based on HNF1 $\alpha$ and KRT81 immunostaining. The HNF $1 \alpha$-positive group showed the best, and KRT81-positive the worst, patient survival (54). In a third unresectable cohort of patients undergoing FOLFIRINOX or gemcitabine therapy, KRT81-positive patients had a negative prognosis compared to $\mathrm{HNF} 1 \alpha$-positive patients, while within the latter group FOLFIRINOX-treated patients displayed a better response than patients under gemcitabine treatment (54).

Lee et al. carried out a genome-wide association study 
to identify candidate single nucleotide polymorphisms (SNP) conferring susceptibility to pancreatic cancer by analyzing almost half a million SNPs from 4,000 pancreatic cancer patients and control subjects (55). This in silico analysis identified 18 candidate SNPs, 11 genes (among them, HNF $1 \alpha$ and HNF4 $\gamma$ ), and 30 pathways that could contribute to pancreatic cancer risk (55).

Some functional studies of HNF1 $\alpha$ have indicated that it can act as a tumor suppressor in PDAC. One recent report showed that siRNA-mediated HNF1 $\alpha$ loss of function caused augmented proliferative capacity and diminished apoptosis (56). In a complementary study, Yu and collaborators explored the role of $\mathrm{HNF} 1 \alpha$ in the regulation of the long non-coding RNA CASC2 (57). HNF1 $\alpha$ activates CASC2 transcription, which in turn can positively regulate PTEN. HNF1 $\alpha$ overexpression in PDAC cell lines induces PTEN expression, decreases p-Akt and p-mTOR and reduces proliferation, while CASC2 knockdown caused the opposite effect (57). In PDAC HNF1 $\alpha$ levels positively correlated with CASC2 expression, and low CASC2 as well as $\mathrm{HNF} 1 \alpha$ levels associated with a poorer patient outcome and shorter overall survival (57).

In contrast, one report has indicated that $\mathrm{HNF} 1 \alpha$ is required for pancreatic CSC function. Specifically, Abel and coworkers showed that HNF1 $\alpha$ and its target gene levels are increased in pancreatic CSC and 3D tumorspheres, while HNF $1 \alpha$ knockdown reduced PDAC growth, apoptosis, tumorsphere formation and pancreatic CSC (58). Notably, HNF1 $\alpha$ silencing in patient derived xenografts hindered tumor progression in vivo (58). Lastly, the authors established an HNF1 $\alpha$-dependent gene signature that correlates with poor patient prognosis (58). The HNF1 $\alpha$ oncogenic role identified in this study is in clear contrast with its tumor suppressive function shown in previous reports $(53,56)$. Different scenarios were discussed as the source of these seemingly antagonistic functions by the authors (58), such as technical differences in virtue of an unusually elevated level of baseline apoptosis found in control cells in Luo et al. (56), differential role of HNF1 $\alpha$ in different PDAC molecular subtypes or the dynamics of its expression as previously identified for PDX1 (59). As discussed below, PDX1 levels and stage specific expression as well as the transcriptional network governed by PDX1 may explain its dichotomous role in pancreatic cancer. Further investigation needs to be done to address whether these discrepancies are reflecting real differential biological properties of HNF $1 \alpha$ in the progression of pancreatic disease.

\section{PDX1}

The homeodomain transcription factor PDX1 is a critical transcriptional regulator involved in development of pancreatic buds and expressed in pancreatic progenitor populations (9). It has fundamental roles in the formation of all pancreatic cell types, as it is expressed throughout the earliest multipotent pancreatic progenitor cell pool, and then in a more restricted manner within all developing and adult islet insulin $+\beta$ cells, where it regulates islet $\beta$ cell function, as well as in a small proportion of islet somatostatin $+\delta$ cells (43). In the adult pancreas, PDX1 levels are elevated in pancreatic regeneration and injury, such as pancreatitis, pancreatic resection and hypoglycemic challenge, where PDX1 expression positively correlates with proliferation of immature progenitor cells $(60,61)$.

IHC analysis of primary human samples has shown that PDX1 is variably expressed in precursor lesions and PDAC. In Park et al.'s report (62), approximately $40 \%$ of PanINs and $35 \%$ of IPMNs exhibited PDX1 expression in at least half of their nuclei. In contrast, only $13 \%$ of PDACs examined exhibited PDX 1 expression in $>50 \%$ of their nuclei, and PDX1 was completely absent in $60 \%$ of PDAC cases (62). This study did not correlate PDX1 levels with grade, and the authors did not observe a significant difference in survival based on PDX1 expression (62).

The functional role of PDX1 in pancreatic malignancy has been extensively reviewed recently $(60,61)$, where the main conclusion was that PDX1 seems to exert primarily oncogenic functions. However, a recent comprehensive analysis of PDX1 in a genetically engineered mouse model demonstrated that this transcription factor has distinct, stage-specific roles in this disease (59). First, the authors ablated a conditional allele of $P d x 1$ in adult murine acinar cells using a tamoxifen-inducible $\mathrm{Cre}^{\mathrm{ER}}$ system. $P d x 1$ deletion enhanced acinar to ductal metaplasia in vivo during acute pancreatitis and in vitro, suggesting that PDX1 plays a role in maintaining acinar cell identity. This was further demonstrated by transcriptomic analysis, which showed that $P d x 1$ deletion impaired the expression of several acinar specific genes in these cells in vitro. Moreover, $P d x 1$ deletion accelerated $\mathrm{Kras}^{\mathrm{G} 12 \mathrm{D}}$-induced acinar to ductal metaplasia and PanIN formation in this mouse model (59). However, inhibition of PDX1 expression in established mouse and human PDAC cell lines generally impaired proliferation and cell survival in vitro and xenograft growth in vivo (59). These results are in consonance with other studies that have suggested that PDX1 has oncogenic properties in 


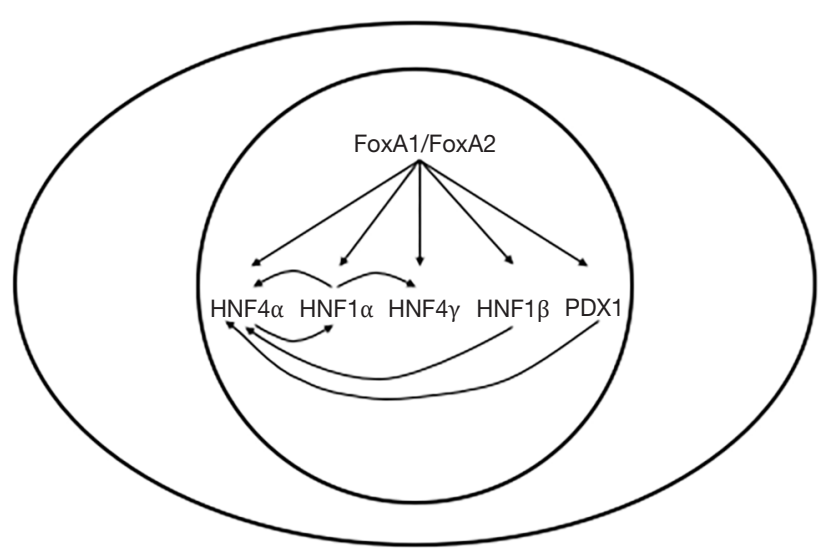

Figure 1 Simplified schematic representation of transcriptional hierarchy and regulatory interplay between pancreatic lineage specifiers (65). The strength of these regulatory interactions appears to vary by cell type.

PDAC $(63,64)$. Using both RNA-Seq and ChIP-Seq, it was shown that PDX1 regulates a distinct set of genes in normal acinar cells compared with established PDAC, which likely accounts for its differential roles in tumor initiation and maintenance (59).

Adding further complexity to the picture, the authors found that low PDX1 expression correlates with reduced survival in two independent patient cohorts comprising all molecular PDAC subtypes identified in Bailey et al. (7) and Collisson et al. (6). These data are in agreement with genomics studies showing that the basal-like/squamous subtype, which has low levels of PDX1, confers a worse prognosis $(5,7)$. To reconcile these correlations with their experimental observations, the authors asked whether a subset of PDAC cells could survive PDX1 inhibition and adapt to a PDX1-low state. Interestingly, they found that approximately 3 weeks after induction of hairpin targeting PDX1, a population of PDX1 low cells emerged with proliferative potential similar to controls (59). These data suggest that the dependence of PDAC cells on PDX1, and likely other lineage specifiers, is not absolute, and that particular selective pressures may lead to the emergence of subpopulations in which downregulation of these lineage specifiers leads to a more poorly differentiated, malignant phenotype.

\section{Conclusions}

Recent high throughput genomic approaches have identified distinct molecular subtypes of pancreatic cancer and relevant molecular players associated with different biological aspects of pancreatic cancer cells. These subtypes are characterized by differential expression of a number of lineage specifiers that play a role in pancreatic development and differentiation (Figure 1). This review presents the work carried out by different groups that collectively deepens our knowledge of the pivotal role that these pancreatic endodermal determinants play in PDAC progression. Several of these lineage specifiers appear to play contextspecific, dichotomous roles in the progression of disease, and additional studies are clearly needed to elucidate the molecular basis of this phenomenon. Moreover, we anticipate that a detailed understanding of these lineage specifiers and their target genes in PDAC will facilitate the identification of new biomarker and therapeutic strategies for this deadly disease.

\section{Acknowledgements}

Funding: EL Snyder was supported in part by a Career Award for Medical Scientists from the Burroughs Wellcome Fund, a V Scholar Award, the Huntsman Cancer Foundation and the NIH (R01CA212415 and funds associated with P30CA042014 awarded to the Nuclear Control Program at the Huntsman Cancer Institute).

\section{Footnote}

Conflicts of Interest: The authors have no conflicts of interest to declare.

\section{References}

1. Oldfield LE, Connor AA, Gallinger S. Molecular Events in the Natural History of Pancreatic Cancer. Trends Cancer 2017;3:336-46.

2. Chiaravalli M, Reni M, O'Reilly EM. Pancreatic ductal adenocarcinoma: State-of-the-art 2017 and new therapeutic strategies. Cancer Treat Rev 2017;60:32-43.

3. Ramos MC, Boulaiz H, Grinan-Lison C, et al. What's new in treatment of pancreatic cancer: a patent review (20102017). Expert Opin Ther Pat 2017;27:1251-66.

4. Pelosi E, Castelli G, Testa U. Pancreatic Cancer: Molecular Characterization, Clonal Evolution and Cancer Stem Cells. Biomedicines 2017;5:E65.

5. Moffitt RA, Marayati R, Flate EL, et al. Virtual microdissection identifies distinct tumor- and stroma- 
specific subtypes of pancreatic ductal adenocarcinoma. Nat Genet 2015;47:1168-78.

6. Collisson EA, Sadanandam A, Olson P, et al. Subtypes of pancreatic ductal adenocarcinoma and their differing responses to therapy. Nat Med 2011;17:500-3.

7. Bailey P, Chang DK, Nones K, et al. Genomic analyses identify molecular subtypes of pancreatic cancer. Nature 2016;531:47-52.

8. Cancer Genome Atlas Research Network. Electronic address aadhe, Cancer Genome Atlas Research N. Integrated Genomic Characterization of Pancreatic Ductal Adenocarcinoma. Cancer Cell 2017;32:185-203.e13.

9. Dassaye R, Naidoo S, Cerf ME. Transcription factor regulation of pancreatic organogenesis, differentiation and maturation. Islets 2016;8:13-34.

10. Vinogradova TV, Sverdlov ED. PDX1: A Unique Pancreatic Master Regulator Constantly Changes Its Functions during Embryonic Development and Progression of Pancreatic Cancer. Biochemistry (Mosc) 2017;82:887-93

11. Zinovyeva MV, Kuzmich AI, Monastyrskaya GS, et al. The role of FOXA subfamily factors in embryonic development and carcinogenesis of the pancreas. Mol Gen Mikrobiol Virusol 2016;34:98-103.

12. Duncan SA, Navas MA, Dufort D, et al. Regulation of a transcription factor network required for differentiation and metabolism. Science 1998;281:692-5.

13. Tomaru Y, Nakanishi M, Miura H, et al. Identification of an inter-transcription factor regulatory network in human hepatoma cells by Matrix RNAi. Nucleic Acids Res 2009;37:1049-60.

14. Wang H, Gauthier BR, Hagenfeldt-Johansson KA, et al. Foxa2 (HNF3beta ) controls multiple genes implicated in metabolism-secretion coupling of glucose-induced insulin release. J Biol Chem 2002;277:17564-70.

15. Li Z, White P, Tuteja G, et al. Foxa1 and Foxa2 regulate bile duct development in mice. J Clin Invest 2009; 119:1537-45.

16. Mishra NK, Guda C. Genome-wide DNA methylation analysis reveals molecular subtypes of pancreatic cancer. Oncotarget 2017;8:28990-9012.

17. Diaferia GR, Balestrieri C, Prosperini E, et al. Dissection of transcriptional and cis-regulatory control of differentiation in human pancreatic cancer. EMBO J 2016;35:595-617.

18. Song Y, Washington MK, Crawford HC. Loss of FOXA1/2 is essential for the epithelial-to-mesenchymal transition in pancreatic cancer. Cancer Res
2010;70:2115-25.

19. Kondratyeva LG, Sveshnikova AA, Grankina EV, et al. Downregulation of expression of mater genes SOX9, FOXA2, and GATA4 in pancreatic cancer cells stimulated with TGFbeta1 epithelial-mesenchymal transition. Dokl Biochem Biophys 2016;469:257-9.

20. Martinelli P, Carrillo-de Santa Pau E, Cox T, et al. GATA6 regulates EMT and tumour dissemination, and is a marker of response to adjuvant chemotherapy in pancreatic cancer. Gut 2017;66:1665-76.

21. Vorvis C, Hatziapostolou M, Mahurkar-Joshi S, et al. Transcriptomic and CRISPR/Cas9 technologies reveal FOXA2 as a tumor suppressor gene in pancreatic cancer. Am J Physiol Gastrointest Liver Physiol 2016;310:G1124-37.

22. Roe JS, Hwang CI, Somerville TDD, et al. Enhancer Reprogramming Promotes Pancreatic Cancer Metastasis. Cell 2017;170:875-88.e20.

23. Santangelo L, Marchetti A, Cicchini C, et al. The stable repression of mesenchymal program is required for hepatocyte identity: a novel role for hepatocyte nuclear factor 4alpha. Hepatology 2011;53:2063-74.

24. Saha SK, Parachoniak CA, Ghanta KS, et al. Mutant IDH inhibits HNF-4alpha to block hepatocyte differentiation and promote biliary cancer. Nature 2014;513:110-4.

25. Yin C, Lin Y, Zhang X, et al. Differentiation therapy of hepatocellular carcinoma in mice with recombinant adenovirus carrying hepatocyte nuclear factor-4alpha gene. Hepatology 2008;48:1528-39.

26. Farkas AE, Hilgarth RS, Capaldo CT, et al. HNF4alpha regulates claudin-7 protein expression during intestinal epithelial differentiation. Am J Pathol 2015;185:2206-18.

27. Wang H, Maechler P, Antinozzi PA, et al. Hepatocyte nuclear factor 4alpha regulates the expression of pancreatic beta -cell genes implicated in glucose metabolism and nutrient-induced insulin secretion. J Biol Chem 2000;275:35953-9.

28. Marcil V, Amre D, Seidman EG, et al. Hepatocyte nuclear factor 4 alpha polymorphisms and the metabolic syndrome in French-Canadian youth. PLoS One 2015;10:e0117238.

29. Winter WE, Nakamura M, House DV. Monogenic diabetes mellitus in youth. The MODY syndromes. Endocrinol Metab Clin North Am 1999;28:765-85.

30. Moore BD, Jin RU, Lo H, et al. Transcriptional Regulation of X-Box-binding Protein One (XBP1) by Hepatocyte Nuclear Factor 4alpha (HNF4Alpha) Is Vital to Beta-cell Function. J Biol Chem 2016;291:6146-57.

31. Tanaka T, Jiang S, Hotta H, et al. Dysregulated expression of $\mathrm{P} 1$ and $\mathrm{P} 2$ promoter-driven hepatocyte nuclear factor- 
4alpha in the pathogenesis of human cancer. J Pathol 2006;208:662-72.

32. Walesky C, Apte U. Role of hepatocyte nuclear factor 4alpha (HNF4alpha) in cell proliferation and cancer. Gene Expr 2015;16:101-8.

33. Bonzo JA, Ferry CH, Matsubara T, et al. Suppression of hepatocyte proliferation by hepatocyte nuclear factor 4alpha in adult mice. J Biol Chem 2012;287:7345-56.

34. Gunewardena S, Walesky C, Apte U. Global Gene Expression Changes in Liver Following Hepatocyte Nuclear Factor 4 alpha deletion in Adult Mice. Genom Data 2015;5:126-8.

35. Sato Y, Hatta M, Karim MF, et al. Anks4b, a novel target of HNF4alpha protein, interacts with GRP78 protein and regulates endoplasmic reticulum stress-induced apoptosis in pancreatic beta-cells. J Biol Chem 2012;287:23236-45.

36. Lu H. Crosstalk of HNF4alpha with extracellular and intracellular signaling pathways in the regulation of hepatic metabolism of drugs and lipids. Acta Pharm Sin B 2016;6:393-408.

37. Matsuo S, Ogawa M, Muckenthaler MU, et al. Hepatocyte Nuclear Factor 4alpha Controls Iron Metabolism and Regulates Transferrin Receptor 2 in Mouse Liver. J Biol Chem 2015;290:30855-65.

38. Babeu JP, Boudreau F. Hepatocyte nuclear factor 4-alpha involvement in liver and intestinal inflammatory networks. World J Gastroenterol 2014;20:22-30.

39. Chahar S, Gandhi V, Yu S, et al. Chromatin profiling reveals regulatory network shifts and a protective role for hepatocyte nuclear factor 4alpha during colitis. Mol Cell Biol 2014;34:3291-304.

40. Kim J, Hoffman JP, Alpaugh RK, et al. An iPSC line from human pancreatic ductal adenocarcinoma undergoes early to invasive stages of pancreatic cancer progression. Cell Rep 2013;3:2088-99.

41. Zhou P, Li B, Liu F, et al. The epithelial to mesenchymal transition (EMT) and cancer stem cells: implication for treatment resistance in pancreatic cancer. Mol Cancer 2017;16:52.

42. Viotti M, Wilson C, McCleland M, et al. SUV420H2 is an epigenetic regulator of epithelial/mesenchymal states in pancreatic cancer. J Cell Biol 2018;217:763-77.

43. Spaeth JM, Gupte M, Perelis M, et al. Defining a Novel Role for the Pdx1 Transcription Factor in Islet beta-Cell Maturation and Proliferation During Weaning. Diabetes 2017;66:2830-9.

44. Noll EM, Eisen C, Stenzinger A, et al. CYP3A5 mediates basal and acquired therapy resistance in different subtypes of pancreatic ductal adenocarcinoma. Nat Med 2016;22:278-87.

45. Kiselyuk A, Lee SH, Farber-Katz S, et al. HNF4alpha antagonists discovered by a high-throughput screen for modulators of the human insulin promoter. Chem Biol 2012;19:806-18.

46. Lee SH, Piran R, Keinan E, et al. Induction of beta-Cell Replication by a Synthetic HNF4 alpha Antagonist. Stem Cells 2013;31:2396-407.

47. Roy N, Malik S, Villanueva KE, et al. Brg1 promotes both tumor-suppressive and oncogenic activities at distinct stages of pancreatic cancer formation. Genes Dev 2015;29:658-71.

48. Wang WJ, Wu MY, Shen M, et al. Cantharidin and norcantharidin impair stemness of pancreatic cancer cells by repressing the beta-catenin pathway and strengthen the cytotoxicity of gemcitabine and erlotinib. Int J Oncol 2015;47:1912-22.

49. Van den Broeck A, Vankelecom H, Van Delm W, et al. Human Pancreatic Cancer Contains a Side Population Expressing Cancer Stem Cell-Associated and Prognostic Genes. PLoS One 2013;8:e73968.

50. Naqvi AAT, Hasan GM, Hassan MI. Investigating the role of transcription factors of pancreas development in pancreatic cancer. Pancreatology 2018;18:184-90.

51. von Wnuck Lipinski K, Sattler K, Peters S, et al. Hepatocyte Nuclear Factor 1A Is a Cell-Intrinsic Transcription Factor Required for B Cell Differentiation and Development in Mice. J Immunol 2016;196:1655-65.

52. Janky R, Binda MM, Allemeersch J, et al. Prognostic relevance of molecular subtypes and master regulators in pancreatic ductal adenocarcinoma. BMC Cancer 2016;16:632.

53. Hoskins JW, Jia J, Flandez M, et al. Transcriptome analysis of pancreatic cancer reveals a tumor suppressor function for HNF1A. Carcinogenesis 2014;35:2670-8.

54. Muckenhuber A, Berger AK, Schlitter AM, et al. Pancreatic Ductal Adenocarcinoma Subtyping Using the Biomarkers Hepatocyte Nuclear Factor-1A and Cytokeratin-81 Correlates with Outcome and Treatment Response. Clin Cancer Res 2018;24:351-9.

55. Lee YH, Gyu Song G. Genome-wide pathway analysis in pancreatic cancer. J Buon 2015;20:1565-75.

56. Luo Z, Li Y, Wang H, et al. Hepatocyte Nuclear Factor 1A (HNF1A) as a Possible Tumor Suppressor in Pancreatic Cancer. PLoS One 2015;10:e0121082.

57. Yu Y, Liang S, Zhou Y, et al. HNF1A/CASC2 regulates pancreatic cancer cell proliferation through PTEN/Akt 
signaling. J Cell Biochem 2017. [Epub ahead of print].

58. Abel A, Goto M, Magnuson M, et al. HNF1A is a Novel Oncogene and Central Regulator of Pancreatic Cancer Stem Cells. bioRxiv 2017. Available online: http://dx.doi. org/10.1101/238097

59. Roy N, Takeuchi KK, Ruggeri JM, et al. PDX1 dynamically regulates pancreatic ductal adenocarcinoma initiation and maintenance. Genes Dev 2016;30:2669-83.

60. Wu J, Liu S, Yu J, et al. Vertically integrated translational studies of PDX1 as a therapeutic target for pancreatic cancer via a novel bifunctional RNAi platform. Cancer Gene Ther 2014;21:48-53.

61. Fendrich V, Lauth $M$. The role of pancreatic and duodenal homeobox 1 as a therapeutic target in pancreatic cancer.

Cite this article as: Camolotto SA, Belova VK, Snyder EL. The role of lineage specifiers in pancreatic ductal adenocarcinoma. J Gastrointest Oncol 2018;9(6):1005-1013. doi: 10.21037/jgo.2018.05.04
Expert Opin Ther Targets 2014;18:1277-83.

62. Park JY, Hong SM, Klimstra DS, et al. Pdx1 expression in pancreatic precursor lesions and neoplasms. Appl Immunohistochem Mol Morphol 2011;19:444-9.

63. Liu SH, Patel S, Gingras MC, et al. PDX-1: demonstration of oncogenic properties in pancreatic cancer. Cancer 2011;117:723-33.

64. Liu SH, Rao DD, Nemunaitis J, et al. PDX-1 Is a Therapeutic Target for Pancreatic Cancer, Insulinoma and Islet Neoplasia Using a Novel RNA Interference Platform. PLoS One 2012;7:e40452.

65. Lau HH, Ng NH, Loo LS, et al. The molecular functions of hepatocyte nuclear factors - In and beyond the liver. J Hepatol 2018;68:1033-48. 